\title{
microRNA regulation of human pancreatic cancer stem cells
}

\author{
Yi-Fan Xu, Bethany N. Hannafon, Wei-Qun Ding \\ Department of Pathology, University of Oklahoma Health Sciences Center, Oklahoma City, Oklahoma, OK 73104, USA \\ Contributions: (I) Conception and design: All authors; (II) Administrative support: None; (III) Provision of study materials or patients: None; (IV) \\ Collection and assembly of data: None; (V) Data analysis and interpretation: None; (VI) Manuscript writing: All authors; (VII) Final approval of \\ manuscript: All authors. \\ Correspondence to: Dr. Wei-Qun Ding. Department of Pathology, University of Oklahoma Health Sciences Center, BRC 411A, 975 NE 10th Street, \\ Oklahoma City, OK 73104, USA. Email: weiqun-ding@ouhsc.edu.
}

\begin{abstract}
RNAs (miRNAs) are a group of small non-coding RNAs that function primarily in the post transcriptional regulation of gene expression in plants and animals. Deregulation of miRNA expression in cancer cells, including pancreatic cancer cells, is well documented, and the involvement of miRNAs in orchestrating tumor genesis and cancer progression has been recognized. This review focuses on recent reports demonstrating that miRNAs are involved in regulation of pancreatic cancer stem cells (CSCs). A number of miRNA species have been identified to be involved in regulating pancreatic CSCs, including miR-21, miR-34, miR-1246, miR-221, the miR-17-92 cluster, the miR-200 and let-7 families. Furthermore, the Notch-signaling pathway and epithelial-mesenchymal transition (EMT) process are associated with miRNA regulation of pancreatic CSCs. Given the significant contribution of CSCs to chemo-resistance and tumor progression, a better understanding of how miRNAs function in pancreatic CSCs could provide novel strategies for the development of therapeutics and diagnostics for this devastating disease.
\end{abstract}

Keywords: Pancreatic cancer; miRNAs; cancer stem cells (CSCs)

Received: 17 November 2016; Accepted: 04 January 2017; Published: 21 January 2017.

doi: $10.21037 /$ sci.2017.01.01

View this article at: http://dx.doi.org/10.21037/sci.2017.01.01

\section{Pancreatic cancer stem cells (CSCs)}

CSCs are cancer cells that possess the ability to self-renew and persist in tumors as a specific cancer cell population that can contribute to relapse and metastasis by re-emerging or by initiating new tumors. The first conclusive evidence showing the existence of CSCs was provided in 1997 by Bonnet and Dick (1). In this study, a subpopulation of leukemia cells expressing CD34, but not CD38 (CD34 ${ }^{+}$ $\mathrm{CD} 38^{-}$), could initiate tumors in NOD/SCID mice that were histologically similar to the donor cells. Cancer stem-like cells were first identified in human cortical glial tumors and were highly similar to neural stem-like cells, but expressed astroglial and neuronal markers (2).

The first pancreatic CSC population was identified in 2007 using xenografts of human pancreatic adenocarcinomas grown in immunocompromised mice. In these mice, a highly tumorigenic subpopulation of pancreatic cancer cells expressing the cell surface markers CD44, CD24, and epithelial-specific antigen (ESA) were isolated (3). It was shown that the CD44(+)CD24(+)ESA(+) subpopulation of cells shared stem cell properties, such as self-renewal, pluripotency, and increased expression of developmental signaling pathways, such as the sonic hedgehog pathway (3). One of the most important aspects of CSC research is the identification of surface markers that can be used to define and isolate CSCs. The widely accepted common CSCs markers are CD44(+) and CD24(+) (4), which are also applicable to pancreatic CSCs. Other surface markers identified for pancreatic CSCs include ABCG2, ALDH1, CD133, c-Met, CXCR4, nestin and nodal-activin (5).

The clinical significance of CSCs has been well described. The concept that CSCs are responsible for the initiation of tumor metastases is supported by the association of CSCs with epithelial-mesenchymal transition (EMT) (6-9). In pancreatic cancer, hedgehog- 
signaling was found to regulate EMT in cancer "stemlike" cells and promote tumor genesis and metastasis (10). A recent report further indicates that exosomes, a group of secreted membrane-bound vesicles, from pancreatic CSCs can reprogram neighboring non-CSCs toward EMT and function in metastatic niche preparation in distant tissues (11). In addition to their role in promoting tumor metastasis, pancreatic CSCs also contribute to chemo-resistance (12). Therefore, targeting CSCs may enhance drug sensitivity and inhibit tumor metastasis (12). Interestingly, CSCs in peripheral blood may also serve as tumor biomarkers for diagnosis or prognosis (13).

\section{microRNAs (miRNAs) and pancreatic CSCs}

miRNAs are short non-coding RNAs (19-22 nucleotides in length) that primarily function to repress target mRNA translation through complementary binding in the $3^{\prime}$ untranslated region (3' UTR) of mRNAs $(14,15)$. miRNAs are transcribed as primary transcripts (pri-miRNA) by RNA polymerase II. The pri-miRNAs are then processed in the nucleus by RNA enzymes into 70-100-nucleotidelong precursors (pre-miRNAs) $(16,17)$. The pre-miRNAs are then translocated to the cytoplasm (18) and further processed by a complex composed of the RNase III enzyme Dicer and the trans-activating response RNAbinding protein (TRBP) $(19,20)$, leading to the generation of the mature miRNA and the consequent degradation of the complementary strands $(21,22)$. The mature miRNAs are loaded onto Argonaute proteins within the RNAinduced silencing complex (RISC) and function to guide the RISC to complementary sequences in the 3' UTR of specific target mRNAs (23-25). Previous studies have shown that some of the miRNAs highly expressed in cancer are oncogenic, such as miR-21, miR-155, miR-175p, miR-19, and miR-92, etc. (26-28); whereas miRNAs with reduced expression in cancer often act as tumor suppressive regulators, such as miR-34, miR-15, miR-16, and let-7, etc. in pancreatic cancer (29-31).

Many studies have shown that miRNAs play a critical role in the regulation of CSCs in malignant tumors, including pancreatic cancer, and are involved in the initiation, propagation, and regulation of EMT and the Notch-signaling pathway in CSCs (32-39). miRNAs that have been reported to regulate pancreatic CSCs include miR-21, miR-34, miR-1246, miR-221, miR-145, the miR-17-92 cluster, and the let-7, miR-200, and miR-30 families.

\section{miRNAs that regulate pancreatic CSCs through the Notch-signaling pathway}

The Notch-signaling pathway is associated with the regulation of cell development in mammary epithelial cells and has been implicated in cancer initiation and progression (40-43). It is deregulated in many kinds of CSCs, including pancreatic CSCs. Recent studies found that Notch-signaling regulates miRNA expression, and in return miRNAs modify Notch-signaling in pancreatic CSCs. For example, overexpression of Notch-1 in pancreatic CSCs led to an increase in expression of miR-21, and a decrease in expression of miR-200b, miR-200c, let-7a, let-7b, and let-7c in vitro (37). Furthermore, metformin, an experimental anticancer drug, decreased the expression of Notch-1, thereby elevating expression of miRNAs such as let-7a, let-7b, miR-26a, miR-101, miR-200b, and miR-200c in a xenograft mouse model (38). With regard to miRNA regulation of Notch signaling, over expression of miR-34 in pancreatic cancer cells either by transfection of miR-34 mimics or infection with lentviral miR-34-MIF led to a significant reduction of cancer initiating cell population likely due to the downregulation of Notch1/2 and Bcl-2 by this miRNA (34). Similarly, DCAMKL-1 (a putative pancreatic stem cell marker) knockdown resulted in down-regulation of Notch-1 expression in a miR-144-dependent mechanism (44). Garcinol, a known plant-derived antioxidant, could downregulate Notch-1 signaling via up-regulation of miR200c, thereby suppressing oncogenic properties of PANC1 cancer stem-like cells (45). Notably, quercetin-induced let-7c was shown to decrease pancreatic cancer initiating cell growth by posttranscriptional activation of Numbl and indirect inhibition of Notch in a fertilized chick egg tumor xenotransplant model (46).

In addition to targeting Notch-signaling, let-7 and miR-34 can regulate pancreatic CSCs through other cellular mechanisms. The let-7 family, which is one of the first discovered miRNAs (47) and the first known human miRNA family (23), was shown to be deregulated in cancerstem-like cells and display tumor suppressor activity (48). In pancreatic cancer, down regulation of let-7 was associated with increased chemotherapy resistance $(49,50)$. In these cells, DCAMKL-1 acted as a master regulator of pancreatic tumor genesis through regulation of multiple tumor suppressor miRNAs including let-7a in a xenograft tumor model (51). Diflourinated-curcumin (CDF) was found to decrease formation of pancreatospheres (spheres generated from pancreatic cancer cell lines' sphere formation assay), 
cell invasiveness, and CSC function in human pancreatic cancer cells by reducing expression of EZH2 and increasing expression of a panel of tumor-suppressive miRNAs, including let-7a-d (52). Treatment of pancreatic CSCs with metformin could decrease the expression of the CSC markers CD44, EpCAM, EZH2, Notch-1, Nanog and Oct4, and induce re-expression of miRNAs (let-7a, let7b, miR-26a, miR-101, miR-200b, and miR-200c) that are typically lost in pancreatic cancer and especially in pancreatospheres (38). Compared to its parental $\mathrm{BxPC}-3$ cells, BxPC-3-LN (highly lymphatic metastatic pancreatic cancer cells derived from $\mathrm{BxP}-3$ cells) cells showed stem cell-like properties, including high lymphatic metastasis potential, self-renewal and chemoresistance (53). The BxPC-3-LN cells also expressed higher levels of migrating CSC surface markers (CD133 and CXCR4) and lower levels of let-7 compared to the parental BxPC-3 cells (53), further supporting the role of let-7 in regulating pancreatic CSCs.

miR-34a is a transcriptional target of p53 and is downregulated in pancreatic cancer (54). For example, highly lymphatic metastatic pancreatic cancer cells possess stem cell-like properties and express low levels of miR-34 in a xenograft model (53). miR-34 was found to be involved in pancreatic CSCs self-renewal by modulation of its downstream targets, Bcl-2 and Notch, implying that miR34 may play an important role in pancreatic CSCs selfrenewal and/or cell-fate determination in vivo (34). The restoration of miR-34 expression by demethylating agent 5-Aza-2'-deoxycytidine (5-Aza-dC) and HDAC inhibitor Vorinostat (SAHA) in CSCs could boost patient response to existing chemotherapies potentially by eliminating the CSC characteristics, which provided mechanistic insight into new therapeutic strategies against pancreatic cancer (54). Another example is that systemic intravenous delivery of miR-34 with nanovectors regulated pancreatic CSCs survival and inhibited growth of $\mathrm{MiaPaCa}-2$ subcutaneous xenografts $(\mathrm{P}<0.01)$. This effect was more pronounced in the orthotropic (intrapancreatic) setting $(\mathrm{P}<0.0005)$ when compared to vehicle controls (55).

\section{miRNAs that regulate EMT in pancreatic CSCs}

EMT refers to a process in which epithelial cells lose their epithelial properties and gain mesenchymal cell characteristics. Cancer cells that undergo EMT acquire stem cell-like properties, thus giving rise to CSCs. The miR-200 family is involved in the regulation of EMT in pancreatic CSCs. Over-expression of miR-200c had an inhibitory effect on human pancreatic CSCs by deregulating EMT-related genes in vitro and in vivo (39). It significantly down-regulated expression of zinc-finger E-box binding homeobox 1 (ZEB1) and vimentin (markers of mesenchymal cells), and up-regulated expression of E-cadherin (marker of epithelial cells), and decreased colony formation, invasion, chemoresistance, and xenograft growth (39). The loss of miR-200a expression was associated with an EMT phenotype and stem-like cell features, characterized by the expression of the cell surface markers CD24, CD44 and ESA in pancreatic cancer cells in vitro (56). Knockdown of DCAMKL-1 up-regulated miR200 expression, resulting in a decrease in the expression of VEGFR1, VEGFR2 and the EMT-related transcription factors ZEB1, ZEB2, SNAIL and SLUG in a xenograft model (51). It was also reported that knockdown of DCAMKL-1 induces miR-200a, along with the downregulation of EMT-associated transcription factors ZEB1, ZEB2, Snail, Slug, and Twist in human pancreatic cancer cells using (44). miR-200 could antagonize EMT driven by mutant KRAS $(57,58)$ and suppression of miR-200 expression by activated oncogenic KRAS promoted cell survival and EMT in KRAS-driven pancreatic cancer in cell lines. Likewise, the activation of Notch-1 signaling contributed to the acquisition of EMT phenotype, which was mediated through miR-200b and CSC self-renewal capacity, and could be attenuated by genistein treatment in vitro (37).

Hypoxia and the HIF pathways also contribute to the acquisition of EMT and maintenance of CSC functions (59). For example, hypoxia could induce mR-21 expression in pancreatic cancer cells via the HIF- $1 \alpha$ pathway. miR21 over-expression in these cells allowed them to escape apoptosis in a hypoxic microenvironment using a xenograft model $(59,60)$. In addition, over-expression of Notch-1 increased expression of miR-21 which led to the acquisition of EMT phenotype in pancreatic cancer cells in vitro (37).

In addition to regulating EMT in pancreatic CSCs, the miR-200 family and miR-21 are also involved in regulating pancreatic CSCs through other mechanisms. The miR-200 family, which consists of miR-200a, miR-200b, miR-200c, miR-141, and miR-429, is involved in cancer metastasis in vitro, as evidenced by a transwell migration assay (61). It was reported that miR-200c overexpression decreases colony formation, invasion and chemoresistance of pancreatic CSCs (62). As described above, CDF, a novel analogue of the turmeric spice component curcumin, decreased pancreatic cancer 
cell survival, clonogenicity, formation of pancreatospheres, and cell invasion. These effects were associated with decreased expression of EZH2 and increased expression of a panel of tumor-suppressive miRNAs, including miR-200b and miR-200c in vitro and in vivo (52). In a xenograft mouse model of human pancreatic cancer, CDF treatment significantly inhibited tumor growth, and was associated with increased miR-200 expression (63). miR-2 1 was highly expressed in pancreatic cancer cell lines, tissues and the plasma of pancreatic cancer patients $(64,65)$ and could promote pancreatic CSCs growth via regulation of FoxO1. FoxO1-negative cells are considered to have CSCs properties in pancreatic cancer and null expression of FoxO1 was associated with a high expression of miR-21 and rapid cell growth in cell lines, primary tumor tissues and a mouse model (66). Moreover, miR-21 expression was related to chemotherapy resistance in pancreatic cancer (49). Inhibition of miR-21 in pancreatic CSCs suppressed tumor genesis, metastasis, and chemotherapy resistance in cell lines (67). CDF treatment of pancreatic cancer in vivo significantly inhibited tumor growth, which was associated with decreased miR-21 expression in tumor remnants (63).

\section{Other miRNAs that are related to pancreatic CSCs}

miR-221 expression was described in pancreatic cancer holoclone-forming cells (a colony-forming stem cell that has a higher growth potential than a meroclone because it does not contain differentiated cells) (68). Inhibiting miR-221 in tumor-initiating stem-like cells could modulate tumor genesis, metastasis, and chemotherapy resistance in pancreatic cancer. The administration of antagomir-221 significantly reduced the stem-like cancer cell fraction, decreased stem-like cancer cell differentiation, thereby reducing chemoresistance to gemcitabine and 5-Fluorouracil in pancreatic cancer cells in vitro and in vivo (67). The miR-17-92 cluster could inhibit tumorigenicity, but enhanced chemoresistance in pancreatic CSCs via the TGF- $\beta 1$ pathway (49). DNMT1 [DNA (cytosine-5)methyltransferase] inhibition was reported to reprogram pancreatic CSCs in part via reactivation of the miR-17-92 cluster in primary tissue cultures and in vivo (69). miR-145 is a tumor suppressor miRNA that could regulate expression of critical pluripotency factors and oncogenes, such as OCT4, SOX2, NANOG, KLF4, KRAS and RREB1, resulting in repressed metastatic potential in pancreatic cancer cells in a xenograft model (51).
miRNA-1246 expression was associated with CCNG2mediated chemoresistance and stemness in pancreatic cancer in primary tissue cultures and an animal model (70). The miR-30 family could promote migratory and invasive characteristics in CD133(+) pancreatic cancer stem-like cells (71). miR-26a expression was reportedly lost in pancreatic cancer and especially in pancreatospheres (38). Finally, miR-99a, miR-100, miR-101, miR-125b, miR192, miR-183 and miR-429 were differentially expressed in pancreatic CSCs compared to non stem-like pancreatic cancer cells $(16,72,73)$.

\section{Future perspectives}

Differential expression of certain miRNA species in pancreatic CSCs and the involvement of these miRNAs in regulation of pancreatic CSCs has been recently documented. These discoveries provide new directions for the development of therapeutics and diagnostics against this malignancy. It remains inconclusive as to whether the miRNA species differentially expressed in pancreatic CSCs are also involved in regulating CSCs in other cancer types. Furthermore, detailed information on how these miRNAs, individually or in combination, regulate pancreatic CSCs thereby contributing to pancreatic cancer progression, merits further exploration. Current management strategies against pancreatic cancer, especially metastatic pancreatic cancer, are not effective. Pancreatic CSCs play a significant role in pancreatic cancer cell self-initiation, metastasis and chemo-resistance, therefore targeting pancreatic CSCs is a promising strategy that could lead to improved pancreatic cancer outcomes.

\section{Acknowledgements}

This work was supported by a grant from Presbyterian Health Foundation.

\section{Footnote}

Conflicts of Interest: The authors have no conflicts of interest to declare.

\section{References}

1. Bonnet D, Dick JE. Human acute myeloid leukemia is organized as a hierarchy that originates from a primitive hematopoietic cell. Nat Med 1997;3:730-7. 
2. Ignatova TN, Kukekov VG, Laywell ED, et al. Human cortical glial tumors contain neural stem-like cells expressing astroglial and neuronal markers in vitro. Glia 2002;39:193-206.

3. Li C, Heidt DG, Dalerba P, et al. Identification of pancreatic cancer stem cells. Cancer Res 2007;67:1030-7.

4. Jaggupilli A, Elkord E. Significance of CD44 and CD24 as cancer stem cell markers: an enduring ambiguity. Clin Dev Immunol 2012;2012:708036.

5. Medema JP. Cancer stem cells: the challenges ahead. Nat Cell Biol 2013;15:338-44.

6. Mani SA, Guo W, Liao MJ, et al. The epithelialmesenchymal transition generates cells with properties of stem cells. Cell 2008;133:704-15.

7. Morel AP, Lievre M, Thomas C, et al. Generation of breast cancer stem cells through epithelial-mesenchymal transition. PLoS One 2008;3:e2888.

8. Scheel C, Eaton EN, Li SH, et al. Paracrine and autocrine signals induce and maintain mesenchymal and stem cell states in the breast. Cell 2011;145:926-40.

9. Brabletz T, Jung A, Spaderna S, et al. Opinion: migrating cancer stem cells - an integrated concept of malignant tumour progression. Nat Rev Cancer 2005;5:744-9.

10. Wang F, Ma L, Zhang Z, et al. Hedgehog Signaling Regulates Epithelial-Mesenchymal Transition in Pancreatic Cancer Stem-Like Cells. J Cancer 2016;7:408-17.

11. Heiler S, Wang Z, Zoller M. Pancreatic cancer stem cell markers and exosomes - the incentive push. World J Gastroenterol 2016;22:5971-6007.

12. Sarkar FH, Li Y, Wang Z, et al. Pancreatic cancer stem cells and EMT in drug resistance and metastasis. Minerva Chir 2009;64:489-500.

13. Lianidou ES, Markou A, Strati A. The Role of CTCs as Tumor Biomarkers. Adv Exp Med Biol 2015;867:341-67.

14. Lin S, Gregory RI. MicroRNA biogenesis pathways in cancer. Nat Rev Cancer 2015;15:321-33.

15. Bartel DP. MicroRNAs: target recognition and regulatory functions. Cell 2009;136:215-33.

16. Bimonte S, Barbieri A, Leongito M, et al. The Role of miRNAs in the Regulation of Pancreatic Cancer Stem Cells. Stem Cells Int 2016;2016:8352684.

17. Lee Y, Ahn C, Han J, et al. The nuclear RNase III Drosha initiates microRNA processing. Nature 2003;425:415-9.

18. Bohnsack MT, Czaplinski K, Gorlich D. Exportin 5 is a RanGTP-dependent dsRNA-binding protein that mediates nuclear export of pre-miRNAs. RNA 2004;10:185-91.

19. Ketting RF, Fischer SE, Bernstein E, et al. Dicer functions in RNA interference and in synthesis of small RNA involved in developmental timing in C. elegans. Genes Dev 2001;15:2654-9.

20. Bernstein E, Kim SY, Carmell MA, et al. Dicer is essential for mouse development. Nat Genet 2003;35:215-7.

21. Uchino K, Takeshita F, Takahashi RU, et al. Therapeutic effects of microRNA-582-5p and $-3 p$ on the inhibition of bladder cancer progression. Mol Ther 2013;21:610-9.

22. Yang $X, D u W W$, Li H, et al. Both mature miR-17-5p and passenger strand miR-17-3p target TIMP3 and induce prostate tumor growth and invasion. Nucleic Acids Res 2013;41:9688-704.

23. Pasquinelli AE, Reinhart BJ, Slack F, et al. Conservation of the sequence and temporal expression of let-7 heterochronic regulatory RNA. Nature 2000;408:86-9.

24. Lee RC, Feinbaum RL, Ambros V. The C. elegans heterochronic gene lin-4 encodes small RNAs with antisense complementarity to lin-14. Cell 1993;75:843-54.

25. John B, Enright AJ, Aravin A, et al. Human MicroRNA targets. PLoS Biol 2004;2:e363.

26. Asangani IA, Rasheed SA, Nikolova DA, et al. MicroRNA-21 (miR-21) post-transcriptionally downregulates tumor suppressor $\mathrm{Pdcd} 4$ and stimulates invasion, intravasation and metastasis in colorectal cancer. Oncogene 2008;27:2128-36.

27. Jiang S, Zhang HW, Lu MH, et al. MicroRNA-155 functions as an OncomiR in breast cancer by targeting the suppressor of cytokine signaling 1 gene. Cancer Res 2010;70:3119-27.

28. Zhang X, Yu H, Lou JR, et al. MicroRNA-19 (miR-19) regulates tissue factor expression in breast cancer cells. $\mathrm{J}$ Biol Chem 2011;286:1429-35.

29. Bae Y, Yang T, Zeng HC, et al. miRNA-34c regulates Notch signaling during bone development. Hum Mol Genet 2012;21:2991-3000.

30. Ji Q, Hao X, Meng Y, et al. Restoration of tumor suppressor miR-34 inhibits human p53-mutant gastric cancer tumorspheres. BMC Cancer 2008;8:266.

31. Zhang B, Pan X, Cobb GP, et al. microRNAs as oncogenes and tumor suppressors. Dev Biol 2007;302:1-12.

32. Liu S, Patel SH, Ginestier C, et al. MicroRNA93 regulates proliferation and differentiation of normal and malignant breast stem cells. PLoS Genet 2012;8:e1002751.

33. Leal JA, Lleonart ME. MicroRNAs and cancer stem cells: therapeutic approaches and future perspectives. Cancer Lett 2013;338:174-83.

34. Ji Q, Hao X, Zhang M, et al. MicroRNA miR-34 inhibits human pancreatic cancer tumor-initiating cells. PLoS One 2009;4:e6816. 
35. Shi Y, Liu C, Liu X, et al. The microRNA miR-34a inhibits non-small cell lung cancer (NSCLC) growth and the CD44hi stem-like NSCLC cells. PLoS One 2014;9:e90022.

36. Bao B, Wang Z, Ali S, et al. Over-expression of FoxM1 leads to epithelial-mesenchymal transition and cancer stem cell phenotype in pancreatic cancer cells. J Cell Biochem 2011;112:2296-306.

37. Bao B, Wang Z, Ali S, et al. Notch-1 induces epithelialmesenchymal transition consistent with cancer stem cell phenotype in pancreatic cancer cells. Cancer Lett 2011;307:26-36.

38. Bao B, Wang Z, Ali S, et al. Metformin inhibits cell proliferation, migration and invasion by attenuating CSC function mediated by deregulating miRNAs in pancreatic cancer cells. Cancer Prev Res (Phila) 2012;5:355-64.

39. Ma C, Ding YC, Yu W, et al. MicroRNA-200c overexpression plays an inhibitory role in human pancreatic cancer stem cells by regulating epithelialmesenchymal transition. Minerva Med 2015;106:193-202.

40. Shimono Y, Mukohyama J, Nakamura S, et al. MicroRNA Regulation of Human Breast Cancer Stem Cells. J Clin Med 2015;5(1). pii: E2.

41. Dontu G, Jackson KW, McNicholas E, et al. Role of Notch signaling in cell-fate determination of human mammary stem/progenitor cells. Breast Cancer Res 2004;6:R605-15.

42. Shi $W$, Harris AL. Notch signaling in breast cancer and tumor angiogenesis: cross-talk and therapeutic potentials. J Mammary Gland Biol Neoplasia 2006;11:41-52.

43. Gangopadhyay S, Nandy A, Hor P, et al. Breast cancer stem cells: a novel therapeutic target. Clin Breast Cancer 2013;13:7-15.

44. Sureban SM, May R, Lightfoot SA, et al. DCAMKL-1 regulates epithelial-mesenchymal transition in human pancreatic cells through a miR-200a-dependent mechanism. Cancer Res 2011;71:2328-38.

45. Huang CC, Lin CM, Huang YJ, et al. Garcinol downregulates Notch1 signaling via modulating miR-200c and suppresses oncogenic properties of PANC-1 cancer stem-like cells. Biotechnol Appl Biochem 2015. [Epub ahead of print].

46. Nwaeburu CC, Bauer N, Zhao Z, et al. Up-regulation of microRNA Let-7c by quercetin inhibits pancreatic cancer progression by activation of Numbl. Oncotarget 2016;7:58367-80.

47. Reinhart BJ, Slack FJ, Basson M, et al. The 21-nucleotide let-7 RNA regulates developmental timing in
Caenorhabditis elegans. Nature 2000;403:901-6.

48. Bao B, Ali S, Ahmad A, et al. Differentially expressed miRNAs in cancer-stem-like cells: markers for tumor cell aggressiveness of pancreatic cancer. Stem Cells Dev 2014;23:1947-58.

49. Ebrahimi S, Ghasemi F, Hassanian SM, et al. Circulating microRNAs as novel potential diagnostic and prognosis biomarkers in pancreatic cancer. Curr Pharm Des 2016. [Epub ahead of print].

50. Long J, Zhang Y, Yu X, et al. Overcoming drug resistance in pancreatic cancer. Expert Opin Ther Targets 2011;15:817-28.

51. Sureban SM, May R, Qu D, et al. DCLK1 regulates pluripotency and angiogenic factors via microRNAdependent mechanisms in pancreatic cancer. PLoS One 2013;8:e73940.

52. Bao B, Ali S, Banerjee S, et al. Curcumin analogue CDF inhibits pancreatic tumor growth by switching on suppressor microRNAs and attenuating EZH2 expression. Cancer Res 2012;72:335-45.

53. Luo G, Long J, Cui X, et al. Highly lymphatic metastatic pancreatic cancer cells possess stem cell-like properties. Int J Oncol 2013;42:979-84.

54. Nalls D, Tang SN, Rodova M, et al. Targeting epigenetic regulation of miR-34a for treatment of pancreatic cancer by inhibition of pancreatic cancer stem cells. PLoS One 2011;6:e24099.

55. Pramanik D, Campbell NR, Karikari C, et al. Restitution of tumor suppressor microRNAs using a systemic nanovector inhibits pancreatic cancer growth in mice. Mol Cancer Ther 2011;10:1470-80.

56. Lu Y, Lu J, Li X, et al. MiR-200a inhibits epithelialmesenchymal transition of pancreatic cancer stem cell. BMC Cancer 2014;14:85.

57. Zhong X, Zheng L, Shen J, et al. Suppression of mir-200 family expression by oncogenic KRAS activation promotes cell survival and epithelial-mesenchymal transition in KRAS-driven cancer. Mol Cell Biol 2016;36:2742-54.

58. Wu X, Wu G, Wu Z, et al. MiR-200a Suppresses the Proliferation and Metastasis in Pancreatic Ductal Adenocarcinoma through Downregulation of DEK Gene. Transl Oncol 2016;9:25-31.

59. Bao B, Ali S, Ahmad A, et al. Hypoxia-induced aggressiveness of pancreatic cancer cells is due to increased expression of VEGF, IL-6 and miR-21, which can be attenuated by CDF treatment. PLoS One 2012;7:e50165.

60. Mace TA, Collins AL, Wojcik SE, et al. Hypoxia induces the overexpression of microRNA-21 in pancreatic cancer 
cells. J Surg Res 2013;184:855-60.

61. Korpal M, Lee ES, Hu G, et al. The miR-200 family inhibits epithelial-mesenchymal transition and cancer cell migration by direct targeting of E-cadherin transcriptional repressors ZEB1 and ZEB2. J Biol Chem 2008;283:14910-4.

62. Ma C, Huang T, Ding YC, et al. MicroRNA-200c overexpression inhibits chemoresistance, invasion and colony formation of human pancreatic cancer stem cells. Int J Clin Exp Pathol 2015;8:6533-9.

63. Bao B, Ali S, Kong D, et al. Anti-tumor activity of a novel compound-CDF is mediated by regulating miR21, miR-200, and PTEN in pancreatic cancer. PLoS One 2011;6:e17850.

64. Abue M, Yokoyama M, Shibuya R, et al. Circulating miR-483-3p and miR-21 is highly expressed in plasma of pancreatic cancer. Int J Oncol 2015;46:539-47.

65. Zhu Z, Gao W, Qian Z, et al. Genetic variation of miRNA sequence in pancreatic cancer. Acta Biochim Biophys Sin (Shanghai) 2009;41:407-13.

66. Song W, Li Q, Wang L, et al. FoxO1-negative cells are cancer stem-like cells in pancreatic ductal adenocarcinoma. Sci Rep 2015;5:10081.

67. Zhao Y, Zhao L, Ischenko I, et al. Antisense inhibition of microRNA-21 and microRNA-221 in tumor-initiating

doi: 10.21037/sci.2017.01.01

Cite this article as: $\mathrm{Xu}$ YF, Hannafon BN, Ding WQ. microRNA regulation of human pancreatic cancer stem cells. Stem Cell Investig 2017;4:5. stem-like cells modulates tumorigenesis, metastasis, and chemotherapy resistance in pancreatic cancer. Target Oncol 2015;10:535-48.

68. Tan L, Sui X, Deng H, et al. Holoclone forming cells from pancreatic cancer cells enrich tumor initiating cells and represent a novel model for study of cancer stem cells. PLoS One 2011;6:e23383.

69. Zagorac S, Alcala S, Fernandez Bayon G, et al. DNMT1 Inhibition Reprograms Pancreatic Cancer Stem Cells via Upregulation of the miR-17-92 Cluster. Cancer Res 2016;76:4546-58.

70. Hasegawa S, Eguchi H, Nagano H, et al. MicroRNA-1246 expression associated with CCNG2-mediated chemoresistance and stemness in pancreatic cancer. Br J Cancer 2014;111:1572-80.

71. Tsukasa K, Ding Q, Miyazaki Y, et al. miR-30 family promotes migratory and invasive abilities in CD133(+) pancreatic cancer stem-like cells. Hum Cell 2016;29:130-7.

72. Jung DE, Wen J, Oh T, et al. Differentially expressed microRNAs in pancreatic cancer stem cells. Pancreas 2011;40:1180-7.

73. Wellner U, Schubert J, Burk UC, et al. The EMTactivator $\mathrm{ZEB} 1$ promotes tumorigenicity by repressing stemness-inhibiting microRNAs. Nat Cell Biol 2009;11:1487-95. 